\title{
Who is really blind in the time of coronavirus: the patient or the doctor? A rare case of Balint's syndrome
}

\author{
Benedetta Storti ${ }^{1,2}$ (i) $\cdot$ Diletta Cereda $^{1} \cdot$ Claudia Balducci $^{1} \cdot$ Francesco Santangelo $^{1,2} \cdot$ Carlo Ferrarese $^{1,2}$. \\ Ildebrando Appollonio ${ }^{1,2}$
}

Received: 12 August 2020 / Accepted: 20 November 2020 / Published online: 5 January 2021

(C) Fondazione Società Italiana di Neurologia 2021

\begin{abstract}
Background Selective bilateral lesions of the parietal-occipital lobes can lead to an uncommon and incompletely understood clinical entity, Balint' syndrome, which consists of simultanagnosia, oculomotor apraxia, optic ataxia and difficulty in perceiving distances between objects.

Case presentation We herein report a rare presentation of Balint's syndrome in a 65-year-old woman suffering from stroke and SARS-CoV2 infection.

Conclusion During SARS-CoV2 pandemic, Italian physicians were forced to work with less instrumental diagnostic resources, relying on their clinical knowledge mostly. The aim of this case report is to highlight the importance of performing a precise neurological evaluation, particularly during these challenging times: it might avoid incorrect diagnosis and favour the discovery of rare clinical diseases.
\end{abstract}

Keywords Balint's syndrome $\cdot$ Stroke $\cdot$ COVID-19 $\cdot$ Cortical blindness

Misdiagnosis is an easy trap during these days of emergency: doctors are forced to visit patients wearing uncomfortable and non-breathable protective suits, and this widens the gap between them and the often aphasic, disoriented, paretic patients suffering a stroke.

Considering the fact that relatives are not allowed into the hospital, it has become extremely challenging to collect a complete medical history of the patient in the emergency room, and it is necessary to communicate over the phone with family members in order to obtain all the essential information about the patient: last known well, any drug allergy, possible contraindication to thrombolysis such as previous cerebral haemorrhage or recent major trauma.

In this scenario TD, a 65-year-old woman had been hospitalized for 1 month due to a severe respiratory failure due to SARS-CoV-2 infection necessitating intubation for several

Benedetta Storti

benedetta.storti@gmail.com

1 Department of Neurology, San Gerardo Hospital ASST, Monza, Italy

2 School of Medicine and Surgery, University of Milano-Bicocca, Monza, Italy days; slowly, she improved, and she was weaned from ventilation support.

One week later, her levels of C-reactive protein and Ddimer were constantly high. A thoracic and abdominal CT was performed discovering a subsegmental pulmonary embolism and several ischemic lesions both in the spleen and in the right kidney. A venous ultrasound was executed, and it showed a deep vein thrombosis in the right iliac veins. Blood testing for thrombophilia, and the research of patent foramen ovale (PFO) with ultrasound scan resulted negative; thus, we speculated that the patient had a diffuse thrombophilia associated with a general state of inflammation caused by SARS-COV2 infection, as previously explained by Ranucci et al. [1].

Moreover, although she was afebrile with vital parameters in the normal range and improving respiratory capability, she appeared persistently disoriented and showed intermittent episodes of decreased consciousness so that a neurologic consult was asked.

On neurologic examination, she was alert, not fully cooperative and disoriented. Motor and sensory examinations showed no clear focal deficit. Language examination was normal too. However, when speaking to the examiner, she always looked at the wrong direction, and when asked to grab a bottle 
Fig. 1 Brain MRI performed at day 32 after admission and showing right frontal and bilateral parietal-occipital lesions. T2 signal is high both in FLAIR (a) and TSE (b) images, suggesting a cerebral ischemia in chronic phase

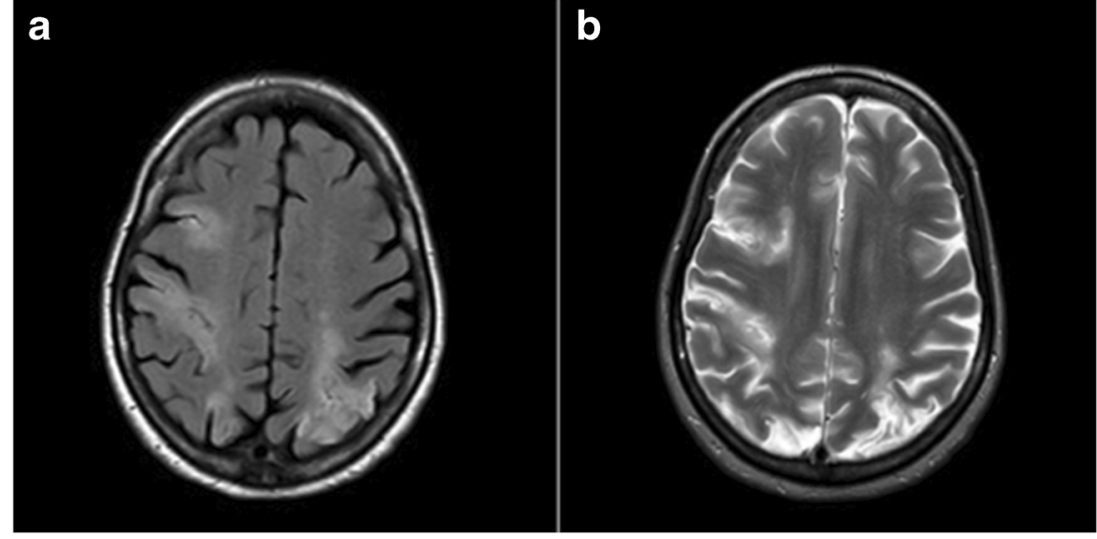

of water kept in front of her, she was not able to do it; furthermore, she was not aware of these visual deficits. Thus, a preliminary hypothesis of cortical blindness was made.

However, her inability to direct the hand to a specific target by using vision and her inappropriate fixation of gaze and difficulties in voluntarily shifting fixation to other objects could also suggest optic ataxia and oculomotor apraxia, respectively, i.e. two key elements for a rare diagnosis of Balint's syndrome [2].

Head CT and afterwards brain MRI (Fig. 1) document right frontal and bilateral parietal-occipital strokes, the latter being the classic lesions of Balint's Syndrome; moreover, the calcarine fissures are both spared, which was against the hypothesis of cortical blindness.

In the following days, we were able to perform additional bedside tests to better verify her visual-spatial performance. When the patient is tested with the Navon figure (Fig.2), which consists of a larger letter composed of copies of a smaller different letter, she saw 'many little S' but was not able to discriminate the larger ' $\mathrm{T}$ ' that those letters were creating.

Thus, cortical blindness could be ruled out, whereas simultanagnosia, the third symptom suggestive for a Balint's

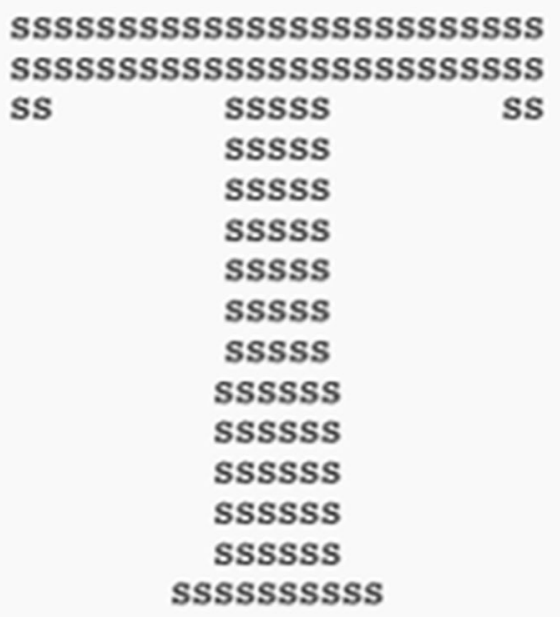

Fig. 2 Navon figure syndrome, was documented: the inability to perceive multiple items in a whole while preserving the ability to recognize single objects.

Finally, also the fourth aspect of Balint's syndrome was documented, i.e. the difficulty in perceiving distances between objects: in fact, when asked which object was nearer between two objects, she always answered with the first named by the examiner.

In this time of health emergency of global concern, it was somewhat unexpected to identify such a clinical rarity as Balint's syndrome.

This case taught us that we should not be overcome by a first impression and driven towards the most intuitive diagnosis. Despite the high risk to be blinded by practical difficulties and lack of means during COVID emergency, the eyes of the clinician should be able to look beyond.

At latest outpatient evaluation, 2 months after discharge, TD appeared dramatically improved: she has recovered completely with only few persisting amnesic episodes.

Data availability The data that support the findings of this study are available from the corresponding author upon reasonable request.

\section{Compliance with ethical standards}

Competing interests The authors declare that they have no competing interests.

Ethical approval None.

\section{References}

1. Ranucci M, Ballotta A, Di Dedda U, Bayshnikova E, Dei Poli M, Resta M, Falco M, Albano G, Menicanti L (2020) The procoagulant pattern of patients with COVID-19 acute respiratory distress syndrome. J Thromb Haemost 18:1747-1751. https://doi.org/10.1111/jth.14854

2. Moreaud O (2003) Balint syndrome. Arch Neurol 60(9):1329-1331

Publisher's note Springer Nature remains neutral with regard to jurisdictional claims in published maps and institutional affiliations. 